\title{
Dissecting the Genetic Architecture of Carbon Partitioning in Sorghum using Multiscale Phenotypes
}

J. Lucas Boatwright ${ }^{1,2, *}$, Sirjan Sapkota ${ }^{1}$, Matthew Myers ${ }^{1}$, Neeraj Kumar ${ }^{1}$, Alex Cox $^{1}$, Stephen Kresovich ${ }^{1,3}$

1 Advanced Plant Technology, Clemson University, 105 Collings Street, Clemson, SC, 29631, USA

2 Plant and Environmental Sciences, Clemson University, 171 Poole Agricultural Center, Clemson, SC, 29631, USA

3 Feed the Future Innovation Lab for Crop Improvement, Cornell University, Ithaca, 14850, NY, USA

\section{* jboatw2@clemson.edu}

\section{Abstract}

Carbon partitioning in plants may be viewed as a dynamic process composed of the many interactions between sources and sinks. The accumulation and distribution of fixed carbon is not dictated simply by the sink strength and number but is dependent upon the source, pathways, and interactions of the system. As such, the study of carbon partitioning through perturbations to the system or through focus on individual traits may fail to produce actionable developments or a comprehensive understanding of the mechanisms underlying this complex process. Using the recently published sorghum carbon-partitioning panel, we collected both macroscale phenotypic characteristics such as plant height, above-ground biomass, and dry weight along with microscale compositional traits to deconvolute the carbon-partitioning pathways in this multipurpose crop. Multivariate analyses of traits resulted in the identification of numerous loci associated with several distinct carbon-partitioning traits, which putatively regulate sugar content, manganese homeostasis, and nitrate transportation. Using a multivariate adaptive shrinkage approach, we identified several loci associated with multiple traits suggesting that pleiotropic and/or interactive effects may positively influence multiple carbon-partitioning traits, or these overlaps may represent molecular switches mediating basal carbon allocating or partitioning networks. Conversely, we also identify a carbon tradeoff where reduced lignin content is associated with increased sugar content. The results presented here support previous studies demonstrating the convoluted nature of carbon partitioning in sorghum and emphasize the importance of taking a holistic approach to the study of carbon partitioning by utilizing multiscale phenotypes.

\section{Introduction}

The integration of multi-scale phenotypes and appropriate mathematical models can assist in the identification of cross-scale interactions leading to emergent properties of dynamic biological systems 1,2]. Indeed, a holistic understanding of complex systems such as plant above-ground biomass and carbon partitioning requires multiscale phenotypes to address changes in anatomical and physiological processes dictated by 
underlying genetic networks [3]. The responsiveness of plant carbon-partitioning regimes to environmental conditions such as those induced by a changing climate can significantly affect crop yields and food security thus requiring attention both regionally [4] and systemically - particularly under conditions of elevated $\mathrm{CO} 2$, heat, drought, and other severe-weather events 8 11]. Crops in the Andropogoneae tribe such as maize [Zea mays (L.)], miscanthus [Miscanthus $x$ Giganteus (Greef et Deuter)], sorghum [Sorghum bicolor (L.) Moench], and sugar cane [Saccharum officinarum (L.)] have been the focus of continued development to serve as staple and/or energy crops under extreme weather conditions $12-16$ and limit ongoing carbon emissions from fossil fuel use $[17-22$. These grasses exhibit highly efficient C4 photosynthetic pathways 23 24, leaf-level nitrogen-use efficiency 25, 26, water-use efficiency 13, 14, 27], and high yields [26, 28].

Sorghum, in particular, is capable of rapidly accumulating significant quantities of carbon and has been designated as an advanced biofuel feedstock by the U.S.

Department of Energy. The Code of Federal Regulations (7 C. F. R. §4288.102) states that advanced biofuels may be derived from biomass in the form of cellulose, hemicellulose, or lignin as well as from sugar or starch [29. Sorghum meets these conditions as it exhibits great diversity in these carbon-partitioning regimes [30], and the sorghum types are further classified based on these traits as cellulosic, forage, grain, or sweet 29 . Sorghum not only meets the requirements as an advanced biofuel feedstock but is capable of rapidly accumulating significant quantities of non-structural [31] and structural carbohydrates [22 32 33] necessary for biofuel [28], forage [34, and grain production [35]. As such, sorghum represents an excellent system for the study of carbon accumulation, partitioning, and designing [29].

Sucrose is the primary source of energy and carbon in plant sink tissues 36 as well as the primary target for ethanol-based, renewable biofuel production [28,37. Synthesis of sucrose occurs in the leaf cytosol after which it is transported to various sinks including both storage sinks (i.e., stems) in addition to structural vegetative and reproductive organs, which function as growth sinks [37 39]. However, changes in the quantities of structural and non-structural carbohydrates do not occur in a one-to-one manner nor are they independent 40,41 . Reduced shoot biomass associated with $d w 3$ has been shown to decrease grain yield via reduced grain size [42, and differences in carbon partitioning in the stem contribute to tradeoffs between structural and non-structural carbohydrate content 31 . Carbon partitioning is also subject to environmental conditions such as those that transition plants between growth and reproductive phases as seen under drought conditions [13. A comprehensive examination of the carbon partitioning sinks is necessary to understand the correlations and tradeoffs between these traits in the form of macroscale phenotyping of traits such as above-ground biomass and plant height to the microscale assessment of compositional traits using tools such as near infrared spectroscopy (NIR) 33, 37, 43.

The sorghum Carbon-Partitioning nested association mapping (CP-NAM) panel 29 contains 11 subpopulations generated using diverse parental accessions from the sorghum Bioenergy Association Panel (BAP) 33 and the recurrent parent, Grassl - an accession capable of accumulating significant biomass and fermentable carbohydrates per unit time and area 44]. NAM populations contain sets of RIL families generated from the diverse founders, and as such, benefit from recombination of the founder genotypes, high allele richness, higher statistical power, and are less sensitive to genetic heterogeneity 29,45. As the CP-NAM covers the diversity of sorghum types and carbon-partitioning regimes, it represents an excellent source of genotypic and phenotypic diversity to elucidate the genetic architecture underlying carbon fixation, translocation, and utilization so that source/sink dynamics and compositional traits may be understood holistically while simultaneously meeting the demands dictated by a 
changing environment 29. Here, we employ quantitative trait locus (QTL) mapping, univariate linear-mixed models (LMMs), and multivariate-response linear-mixed models (MV-LMMs) to identify loci associated with the primary carbon sinks represented by structural and non-structural carbohydrate content in sorghum. Using publicly available genomic resources from the sorghum CP-NAM [29], we identify numerous putative loci involved in carbon partitioning both known and novel.

\section{Materials and Methods}

\section{Plant Materials and Phenotyping}

CP-NAM seeds were accessed from the Clemson University sorghum germplasm collection and planted at the Simpson Research Farm (34.64737384683981, -82.74780269784793), South Carolina in May of 2020. While the original CP-NAM panel contained 2,489 accessions, a subset of 110 individuals were selected from each RIL family using the Partitioning Around Medoids (PAM) function in the R package cluster 46 to reduce the field size and manual labor necessary to manage the CP-NAM field while representing most of the genetic diversity within the RIL populations [47. A total of 110 sample clusters were identified based on the genomic data from each RIL family with a medoid sample centrally located in each cluster (Figure 1). The medoid is the individual within the cluster that best represents the genetic diversity of that sample cluster. The 110 accessions representing the medoids for each population were selected as representatives of each cluster and planted for phenotyping in 2020.

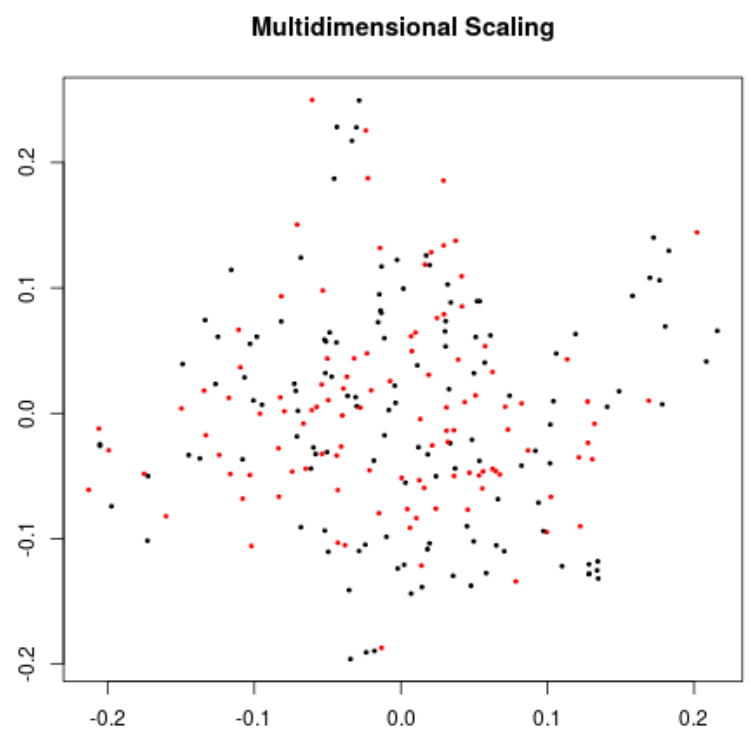

Figure 1. A multidimensional scaling plot using the genotypic data of PI229841 RIL accessions where the 110 medoid samples are colored red and the remaining samples are black. The x-axis represents the first principal component of the genotypic data, and the y-axis represents the second principal component. The proximity of accessions to each other indicates the degree of similarity - with shorter distances indicating higher similarity.

The NAM RILs were planted in single-row plots that were $3.04 \mathrm{~m}$ in length with 
$0.762 \mathrm{~m}$ between-row spacing in a randomized complete block design with two replicates per line. Randomization was done within blocks containing RILs from a given family, and families were planted together to avoid large field effects within families. Plants were irrigated on an as-needed basis but did not occur past 90 days after planting due to plants exceeding the irrigation pivot height. Harvesting started September 14th and continued through October 19th. Due to the range in harvesting days, the maturity stage and days to harvest were recorded for each plot to use as covariates for phenotypic and genomic analysis to avoid the confounding effect of varying maturity groups across the RIL families. Phenotypes collected included above-ground biomass, stand count, maturity at harvest, days to harvest (DTH), plant height, and dry and wet weights. Stand count was measured as the total number of emerged seedlings between 15-30 days after planting. Plant height was measured at harvest from the base of the stalk to the apex of the panicle, or, if no panicle was present, to the apex of the shoot apical meristem.

A representative meter was selected for each plot, and all stalks were cut at the base within that meter and weighed (in kilograms). From those stalks, three representatives were selected for subsequent phenotyping including above-ground biomass (including panicles and leaves), wet weight, and dry weight, where biomass represents a scaled meter weight. Any panicles or partially formed panicles were removed along with all leaf matter before collecting wet weight. The stalks were then cut into billets for collection into mesh drying bags and placed into drying bins at $40{ }^{\circ} \mathrm{C}$ until stalks were dried to a constant moisture content. Dried stalks were removed from the drying bins, and dry weights were taken. Stalks were then ground with a Retsch SM 300 cutting mill so that compositional traits could be measured using a PerkinElmer DA7250 ${ }^{\mathrm{TM}} \mathrm{NIR}$ instrument (https://www.perkinelmer.com), which uses calibration curves for spectral measurements built using wet chemistry values generated by Dairyland Laboratories, Inc. (Arcadia, WI, USA) as described in (Brenton2016-lf). Compositional traits include acid detergent fiber (ADF), adjusted crude protein (Adj. CP), neutral detergent fiber (NDF), ash-free NDF (aNDFom), ash, calcium, chloride, dietary cation-anion difference (DCAD), dry matter, potassium, lignin, magnesium, moisture, net energy growth (NEG), net energy lactation (NEL), relative feed value (RFV), non-fiber carbohydrates (NFC), and water-soluble carbohydrates (WSC) where all traits are expressed as a percent of dry matter. NEG, NEL, NEM, and TDN were also estimated using an Ohio Agricultural Research \& Development Center (OARDC) summative energy equation and may appear conjugated with the OARDC abbreviation (Figure 2).

\section{Descriptive Statistics and QTL Mapping}

The repeatability was estimated for all traits using the package heritability in the $\mathrm{R}$ programming language ( $\mathrm{R}$ Core Team 2019) [49]. The best linear unbiased predictors (BLUPs) were calculated for each trait with the R package lme4 [50] using the lmer function with genotype as random effects. The resulting BLUPs were used as adjusted phenotypic values for all mapping and association analyses. Heatmaps and correlation metrics were measured using the seaborn [51] and pandas [52] packages in CPython [53], respectively.

Genotypic data from 29] were used to perform QTL mapping and genome-wide association studies (GWAS). In summary, Genotyping-By-Sequencing (GBS) data were generated using a double-digest approach ( PstI and MspI), processed using the Tassel GBS version 2 pipeline [54], and imputed using Beagle 5.1 [55] as described in [29]. Genetic maps were constructed for each RIL family with Haldane's mapping function 56] and a genotyping error rate of 0.0001 , where the conditional probabilities of the true genotypes were estimated using a hidden Markov model. Map construction and QTL mapping were performed for each RIL family using the qtl2 package [57] in R, 


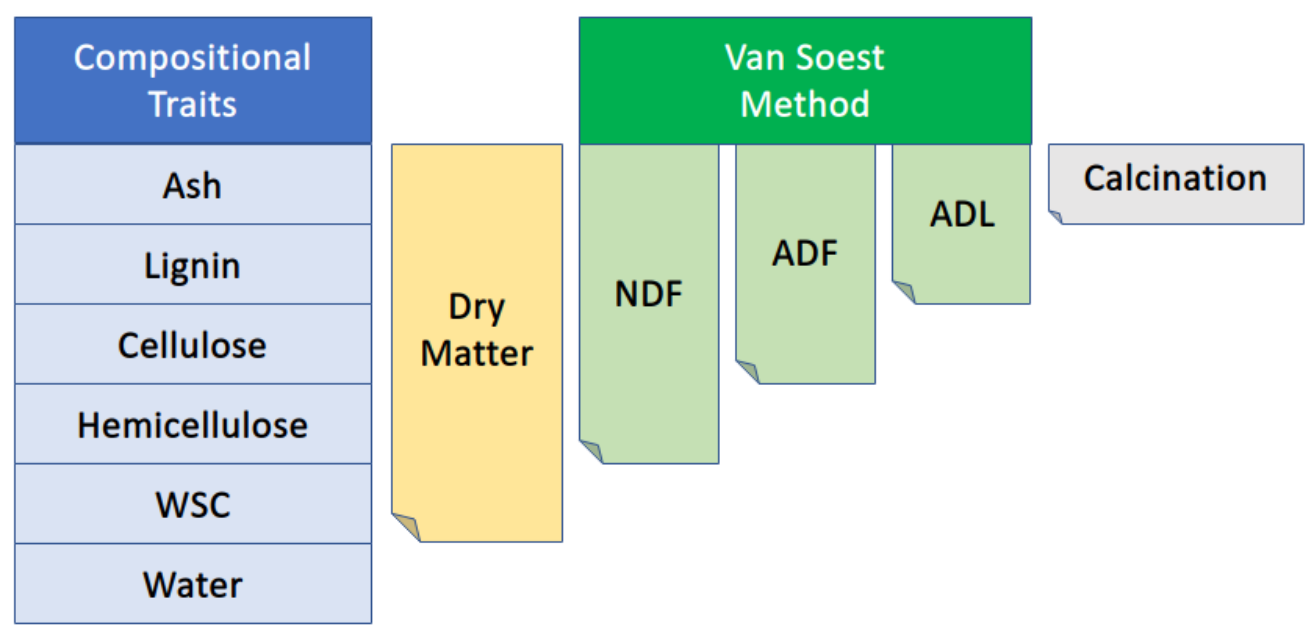

Figure 2. Diagram of the relationship among several compositional traits collected via NIR. NDF, neutral detergent fiber; ADF, acid detergent fiber; ADL, acid detergent lignin; WSC, water-soluble carbohydrates. The Van Soest method permits the distinction of soluble cell contents from the less digestible cellular components such as lignin, cellulose, and hemicellulose. Figure adapted from 48 .

and genomic scans were performed using Haley-Knott regression [58] and linear mixed model approaches [57] including both full and leave-one-chromosome-out models. All models included maturity and DTH as covariates except for DTH. QTL effects were estimated as $100 \times\left(1-10^{(-2 \times L O D) / n}\right)$, where $n$ is the number of individuals in the corresponding mapping population [59]. However, we recognize that estimates for PVE with these population sizes may exhibit inflated values due to the Beavis effect 60 .

\section{Genome-Wide Association}

GWAS were done using both the Genome-wide Efficient Mixed Model Association (GEMMA) program version 0.98.3 [61] and the Genome Association and Prediction Integrated Tool (GAPIT) version $3[62,63$. We specifically used GEMMA for both MV-LMMs and Bayesian Sparse Linear Mixed Models (BSLMM) 64 while GAPIT was used for BLINK [65] and LMMs. The phenotypic and genotypic data were converted to Plink format using Plink [v1.90b6.10] 66. The genomic relatedness matrix was calculated using the VanRaden algorithm 67 and all models were run using a MAF filter of 0.01. Univariate LMMs are of the following the form,

$$
\begin{gathered}
y=\mathbf{X} \beta+\mathbf{Z} u+\epsilon ; \\
u \sim N(0, \mathbf{G}) \text { and } \epsilon \sim N(0, \mathbf{R}),
\end{gathered}
$$

131

where $\mathbf{y}$ is a vector of phenotypic values for a single trait, $\mathbf{X}$ is the numeric genotype matrix generated from the SNPs, $\beta$ represents the unknown vector of fixed effects representing the effect size for each SNPs, $\mathbf{Z}$ is the design matrix for random effects, $\mathbf{u}$ is the unknown vector of random effects, and $\epsilon$ is the unknown vector of residuals. These univariate models test the alternative hypothesis $H_{1}: \beta \neq 0$ against the null hypothesis $H_{0}: \beta=0$ for each SNP.

In addition to the frequentist univariate model described above which estimates fixed effect coefficients by selecting the optimal value minimizing the least-squared error - the equivalent of a flat prior, we ran a BSLMM which assumes fixed effects are 
distributed according to the sparse prior, $\beta \sim \pi N(0, \sigma 2 a \tau-1)+(1-\pi) \delta_{0}$ [64]. Runs were executed using 20e6 sampling steps with a burn-in of 5e6, and the posterior inclusion probability (PIP) threshold established as 0.036 based upon a $99.95 \%$ quantile from simulated data sets across quantiles to determine the empirical significance cutoff 68. While more computationally intensive due to the Markov Chain Monte Carlo sampling approach involved, this model provides shrinkage of $\beta$ estimates to control for type I errors and provides a posterior distribution of plausible values rather than simple point estimates. Additionally, we also conducted univariate analyses using BLINK 65. BLINK approximates the maximum likelihood approach used by LMMs, instead using Bayesian Information Criteria in a fixed effect model where each SNP is iteratively associated with the phenotype of interest. Markers in linkage disequilibrium (LD) with the most significant marker are then excluded - as estimated using a Pearson correlation coefficient $\leq 0.7$. For subsequent markers, the next most significant SNP is selected, and the exclusion process is conducted in the same way until no markers can be excluded. Unlike SUPER and FarmCPU methods, BLINK does not assume that causal genes are evenly distributed across the genome and is faster with higher statistical power - due to its multi-locus approach - and lower type I error rates 65].

For MV-LMMs, we used GEMMA models of the form:

$$
\begin{gathered}
\mathbf{Y}=\mathbf{X} \beta+\mathbf{Z} \mathbf{U}+\mathbf{E} ; \\
\mathbf{U} \sim M V N_{n \times d}\left(0, \mathbf{K}, V_{g}\right), \\
\mathbf{E} \sim M V N_{n \times d}\left(0, I_{n \times n}, V_{e}\right),
\end{gathered}
$$

where $\mathbf{Y}$ is an $\mathrm{n}$ by $\mathrm{d}$ matrix of $\mathrm{d}$ phenotypes for $\mathrm{n}$ individuals, $\mathbf{X}$ is the numeric genotype matrix generated from the SNPs, $\beta$ is a $d$ vector of fixed effects representing the effect size for the d phenotypes, $\mathbf{Z}$ is the design matrix for random effects, $\mathbf{U}$ is the $\mathrm{n}$ by $\mathrm{d}$ matrix of random effects, $\mathbf{E}$ is the $\mathrm{n}$ by $\mathrm{d}$ matrix of residuals, $\mathbf{K}$ is a known $\mathrm{n}$ by $\mathrm{n}$ relatedness matrix, $V_{g}$ is a d by d symmetric matrix of genetic variance components, $I_{n \times n}$ is an $\mathrm{n}$ by $\mathrm{n}$ identity matrix, and $V_{e}$ is a $\mathrm{d}$ by $\mathrm{d}$ symmetric matrix of environmental variance components. As the maturity of accessions significantly affects all phenotypes, maturity and DTH were used as covariates in all QTL mapping and GWAS models except for DTH.

\section{Multivariate Adaptive Shrinkage}

The estimated effect sizes and standard errors for every marker in the the LMMs for ADF, ash, dry matter, dry weight, height, NDF, P, wet weight, and WSC were filtered using a local false sign rate $<0.1$ based on a condition-by-condition analysis using ashr in $\mathrm{R}$ 69]. A control set of estimated effects and standard errors were also randomly selected for 40,000 markers to estimate the covariance between SNPs for each phenotype. A correlation matrix of the random control set was estimated and used to control for the confounding effects of correlated variation among the traits using mashr in $\mathrm{R}$ [70]. We utilized both canonical and data-driven covariance matrices following mashr best practices to test for pleiotropy across traits 70 . The posterior probabilities were calculated for each SNP by fitting a mash model with all tests. Bayes factors were extracted and plotted from mash results using the CDBNgenomics R package 71]. Variants exhibiting Bayes Factors greater than 10 were considered as demonstrating significant pleiotropic effects. 
Table 1. Repeatability for agronomic (top portion of table) and compositional (bottom portion) traits. Agronomic traits include maturity at harvest, plant height, wet weight, dry weight, above-ground biomass, and days to harvest. Compositional traits include acid detergent fiber (ADF), adjusted crude protein (Adj. CP), ash-free neutral detergent fiber (aNDFom), crude protein, dry matter, lignin, neutral detergent fiber (NDF), net energy growth (NEG), total digestible nutrients (TDN) as a percentage of ADF, and water-soluble carbohydrates (WSC). NEG was estimated using an Ohio Agricultural Research \& Development Center (OARDC) summative energy equation.

\begin{tabular}{cc} 
Trait & Repeatability \\
\hline Maturity & 0.81 \\
Plant Height & 0.53 \\
Wet Weight & 0.52 \\
Dry Weight & 0.55 \\
Biomass & 0.52 \\
DTH & 0.76 \\
\hline ADF & 0.65 \\
Adj. CP & 0.38 \\
aNDFom & 0.61 \\
Crude protein & 0.35 \\
Dry Matter & 0.36 \\
Lignin & 0.45 \\
NDF & 0.55 \\
NEG (OARDC) & 0.59 \\
WSC & 0.47 \\
TDN (ADF) & 0.62
\end{tabular}

\section{Results}

\section{Trait Heritabilities and Correlations}

Heritability is the proportion of phenotypic variance attributable to genetic variance, and when differences between genotypes is assumed to derive entirely from genetic effects, the measurement of consistent individual differences is called repeatability [49]. We calculated the repeatability for all traits and identified many traits with repeatability greater than 0.2 (Table 1) with maturity and DTH exhibiting the highest repeatabilities $(>0.75)$. Agronomic phenotypes exhibited higher repeatability compared to compositional traits with all agronomic traits exceeding 0.5 repeatability. Agronomic traits also demonstrated relatively low correlation among the traits except for biomass, wet weight, and dry weight which were all highly correlated $(\geq 0.67)$ (Figure 3). These measures for repeatability and correlation among traits are consistent with previous estimates in sorghum 33.

Many compositional traits exhibited strong correlation $(>|0.5|)$ (Figure 4), which is expected due to the aggregate nature of some traits and their dependency on maturity (Figure 2). Importantly, while many of the fiber-based compositional traits exhibited strong repeatability, only six compositional traits of 34 had Pearson's correlation coefficients $>|0.3|$ with dry weight and none had values exceeding $|0.5|$. The lack of strong correlation between compositional traits and dry weight suggests that sorghum composition could be improved without significantly affecting total vegetative yield 33 . 43 . 


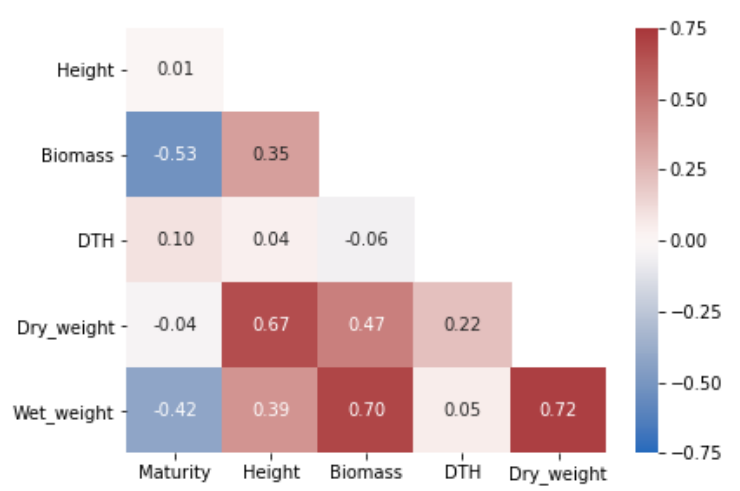

Figure 3. Heatmap of Pearson's correlation coefficients among agronomic phenotypes. Biomass represents a scaled meter weight, and DTH is days to harvest.

\section{Mapping and Associations}

\section{Agronomic Traits}

As the CP-NAM is composed of 11 RIL families, it provides the opportunity to resolve genotype-to-phenotype associations through both QTL mapping and GWAS. To this end, QTL mapping was performed for each RIL family in the CP-NAM for every trait using maturity and DTH as covariates except when DTH is the response variable. Several known QTL were identified for plant height on chromosomes (Chr) 6 [qHT\%.1/Dw2], Chr7 [Dw3], and Chr9 [Dw1] aggregated by RIL families derived from grain, cellulosic, sweet, and forage parents (Figure 5. Table S2) along with several potentially novel associations on Chr1 and Chr8. The newly identified QTL on Chr1 spanned from 10 to $12 \mathrm{Mb}$ (13.2 PVE) and from 22 to $56.7 \mathrm{Mb}$ (14.5 PVE) (Table QtlAgronomic). The QTL from the latter position also had a significant genome-wide association for height using the BLINK model with a peak at Chr1:50,888,855 (Figure S1). Another novel QTL was identified for height on Chr8 from approximately 0.37 to $2.7 \mathrm{Mb}$ (Table Chr8Height) using a leave-one-chromosome-out method (Figure 5; ; Figure S2). A significant genome-wide association for height was found for the SNP at Chr8:2,033,695 using the BSLMM model (Figure S3), and the associated region is within previously identified QTL for transpiration rate and efficiency of energy of PSII 72].

Sorghum has over 40 identified flowering time and maturity QTL 73. QTL mapping results for DTH in PI506069 RILs identified a locus on Chr4 (11.8 PVE) from 70 to 113 $\mathrm{cM}$ that peaked at $79 \mathrm{cM}$ (Figure 5b). This QTL colocalizes with the flowering time gene CN2 [74, which is a centroradialis-like gene homologous to Terminal Flower1 (TFL1). An additional 11 loci were identified using BLINK including Ma3/Ma5 [Chr1], SbCN12 [Chr3], and Ma1 [Chr6] 75] along with several other unidentified loci (Figure S4). The identified loci include phytochromes and other flowering time modulators that mediate photoperiod sensitivity in these non-temperately adapted accessions.

\section{Biomass Traits}

In addition to height and DTH phenotypes, various measures of biomass yield were taken including wet weight, dry weight, and above-ground biomass (abbreviated as biomass). These biomass traits were often associated with the same QTL - particularly the QTL on Chr6 and Chr7 (Figure 5), but significant associations from GWAS were more variable (Figure 6). The QTL on Chr3 (12.7 PVE) identified using wet weight spans from approximately 1 to $6 \mathrm{Mb}$ in the sweet $\mathrm{x}$ cellulosic RILs of PI586454 (Figure 5F) and coincides with QTL associated with stem circumference and transpiration 


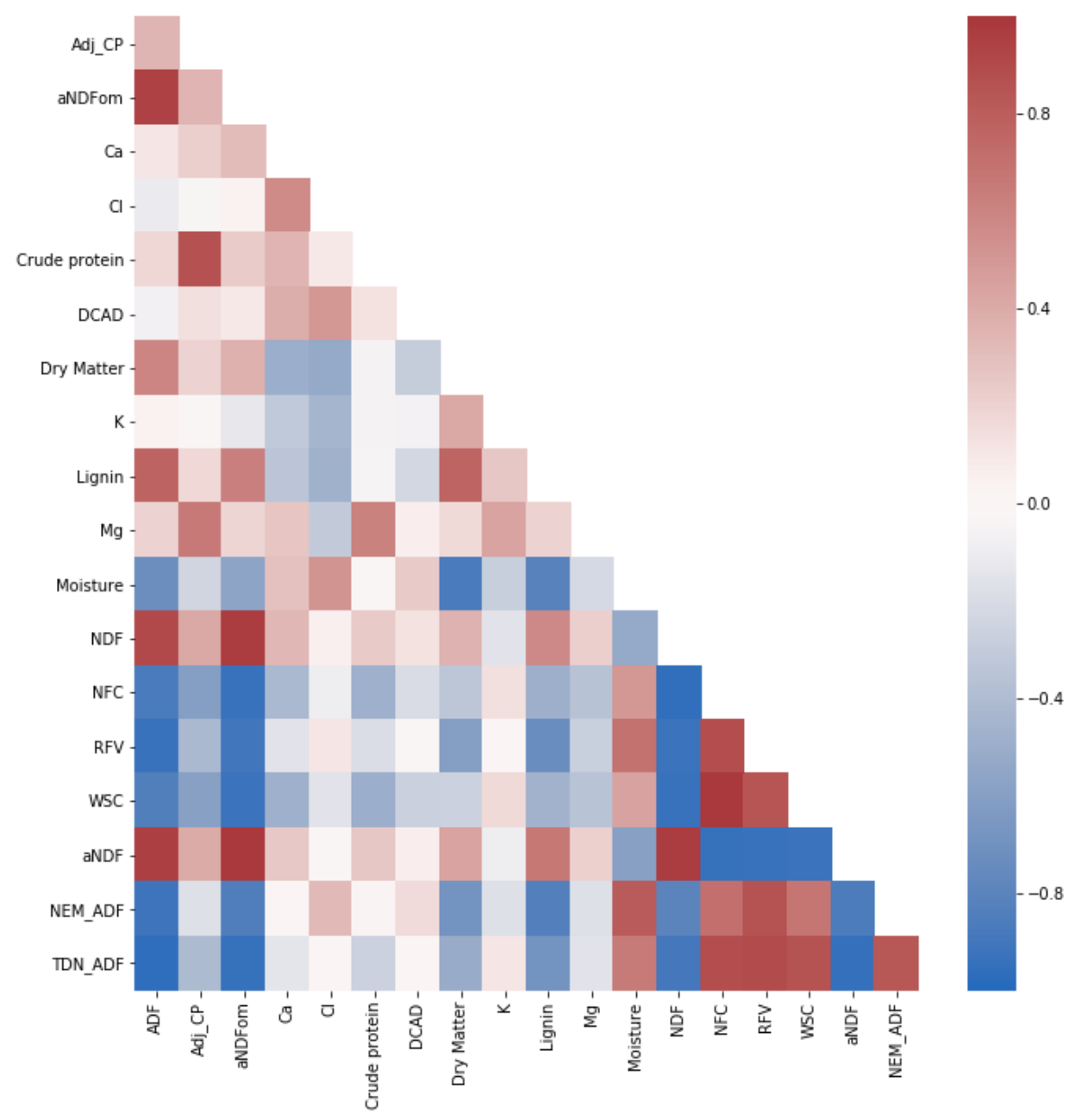

Figure 4. Heatmap of Pearson's correlation coefficient among compositional traits. Traits include acid detergent fiber (ADF), adjusted crude protein (Adj.CP), neutral detergent fiber (NDF), ash-free NDF (aNDFom), calcium, chloride, crude protein, dietary cation-anion difference (DCAD), dry matter, potassium, lignin, magnesium, moisture, non-fiber carbohydrates (NFC), relative feed value (RFV), and water-soluble carbohydrates (WSC). NEG, NEL, NEM, and TDN were also estimated using an Ohio Agricultural Research \& Development Center (OARDC) summative energy equation and may appear conjugated with the OARDC abbreviation. 
(a)

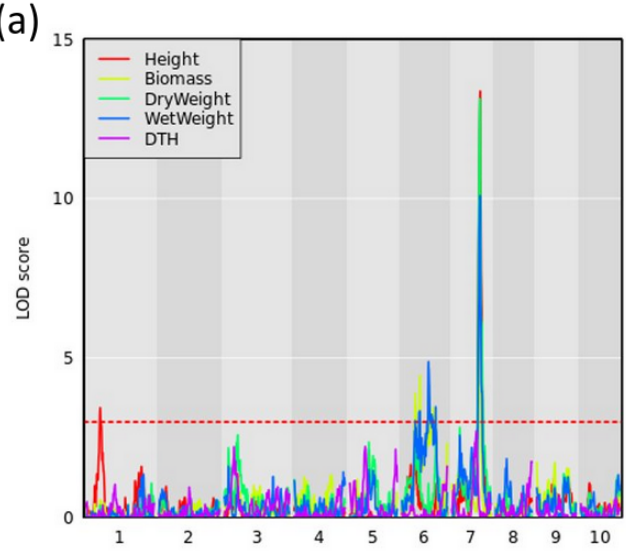

(c)

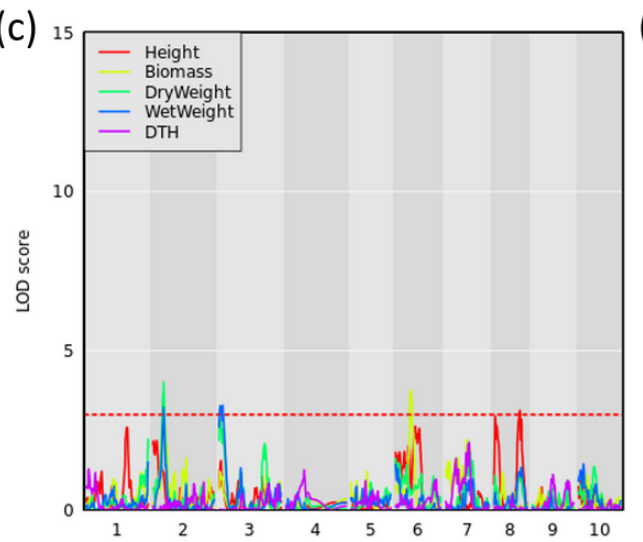

(b)

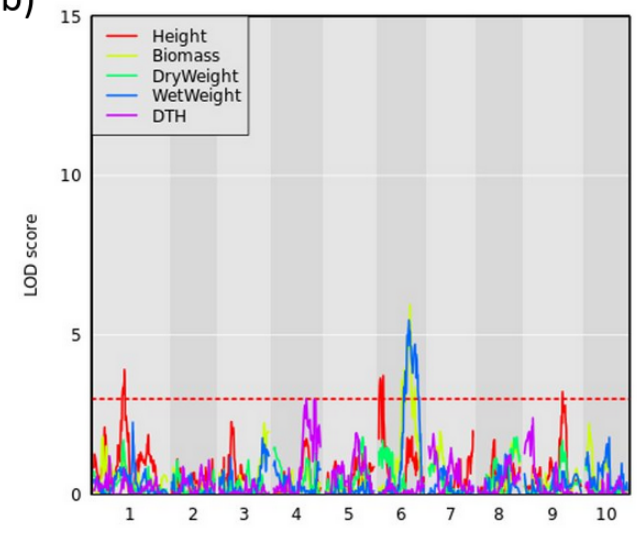

(d)

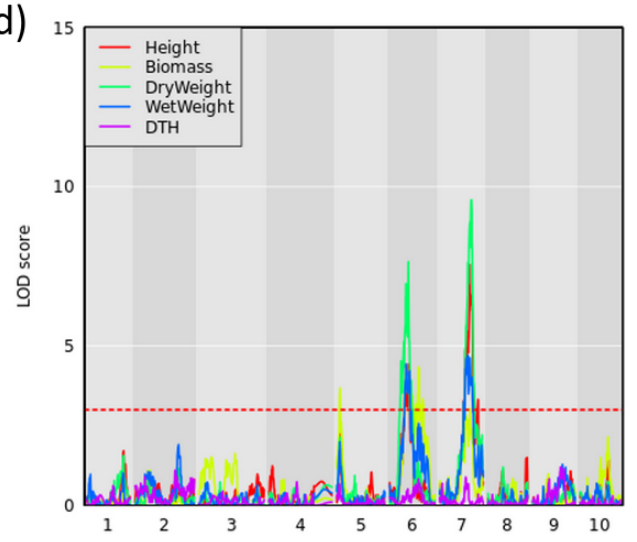

Figure 5. QTL mapping for agronomic traits with maturity covariate where the red dashed line represents a logarithm of the odds (LOD) threshold of three in (a) PI297155, (b) PI506069, (c) PI586454, and (d) PI655972 RILs, which are grain, cellulosic, sweet, and forage recombinant populations, respectively. 
rate 72,76. GWAS of wet weight also identified an association on Chr3 at approximately $62 \mathrm{Mb}$ (Figure 6 $\mathrm{d}$ ), which colocalizes with numerous trait associations including plant height [77, stem circumference [76], and days to flowering [78. However, the gene(s) mediating these phenotypes is unclear. Dry weight and wet weight were associated with several QTL on Chr6 (Figure $5 \mathrm{a}$, d) that were also captured through GWAS (Figure 6) and ranged from 1 to $5 \mathrm{Mb}$ and 49 to $51 \mathrm{Mb}$, respectively. The QTL spanning 1 to $5 \mathrm{Mb}$ corresponds to the known maturity locus, Ma6 [79. These phenotypes also captured the height loci Dw2, which encodes a protein kinase that regulates stem internode length [80, and $D w 3$, which encode a P-glycoprotein auxin transporter and only affects plant height below the flag leaf [81]. As auxin stimulates the production of hemicellulose and consequently stem elongation, the association is consistent with the known identity of the locus 81].

The forage RIL family, CP-NAM PI655972, was uniquely suited for identifying a QTL (14.3 PVE) controlling biomass content on chromosome 5 (Figure 5d). The biomass QTL also overlapped a QTL for adjusted crude protein content (Table QTLCompositional). 82 identified a sucrose content QTL that falls completely within the biomass QTL and partially overlaps the adjusted crude protein QTL seen here 82 . Given the large range of the QTL or even the overlapping region, it is difficult to pin down what gene(s) may be responsible for these associations. In addition to this unique locus, biomass was associated with the same loci on Chr6 and Chr7 as height, wet weight, and dry weight (Figure 6).

\section{Compositional Traits}

QTL mapping was also performed for all compositional traits, and select traits were plotted for all RIL families (Figure 7). Several overlapping QTL were identified across traits within RIL families, and the various sorghum RIL families/types captured different QTL for the same traits. The most significant QTL on Chr6 associated with ADF was consistently identified in all RIL families. The narrowest range of this locus was obtained in PI229841 and PI508366 RILs and spanned from approximately 50.3 to $51.8 \mathrm{Mb}$ (Table QtINIRTable).

WSC content provides an estimate of the carbon partitioned and accumulated by accessions in the stem in the form of water-soluble carbohydrates [37. For WSC, we identified a QTL on Chr6 in the PI22913 RILs around $50 \mathrm{Mb}$, which results from a cross between the sweet sorghum accession PI22913 and the cellulosic Grassl (Figure 7a). The QTL occurs within the Dry Midrib $(D)$ locus, which has also shown strong association with midrib color, grain yield, sugar yield, juice volume, and biomass, indicative of a pleiotropic effect of the $D$ locus across these phenotypes 83. 84]. This QTL also overlaps with QTL identified using ADF, wet weight and dry weight phenotypes described above. 83 previously demonstrated that green midrib color was more strongly associated with sugar content traits than the $D$ locus genotypic data with sugar content, and therefore suggested that selecting for green midribs is a simple alternative to genetic selection for sweet sorghum breeding programs. Consistent with this observation, the $D$ locus accounted for $64.2 \%$ of the variance explained for WSC in PI229841 RILs.

Following the design pattern indicated in Figure 2, we used a combination of LMMs with various compositional traits as covariates to deconvolute the contribution of individual traits to phenotypic variance, and as a converse approach, we also ran multivariate-response models on constituent parts to compare with composite traits. While population genomic studies have historically utilized univariate LMMs, more recent works are finding that multivariate-response linear mixed models (MV-LMM) have higher true-positive rates particularly when correlated traits with low, medium, and high heritabilities are analyzed together in one MV-LMM [85]. The use of MV-LMMs may also provide additional power to detect causal loci exhibiting 


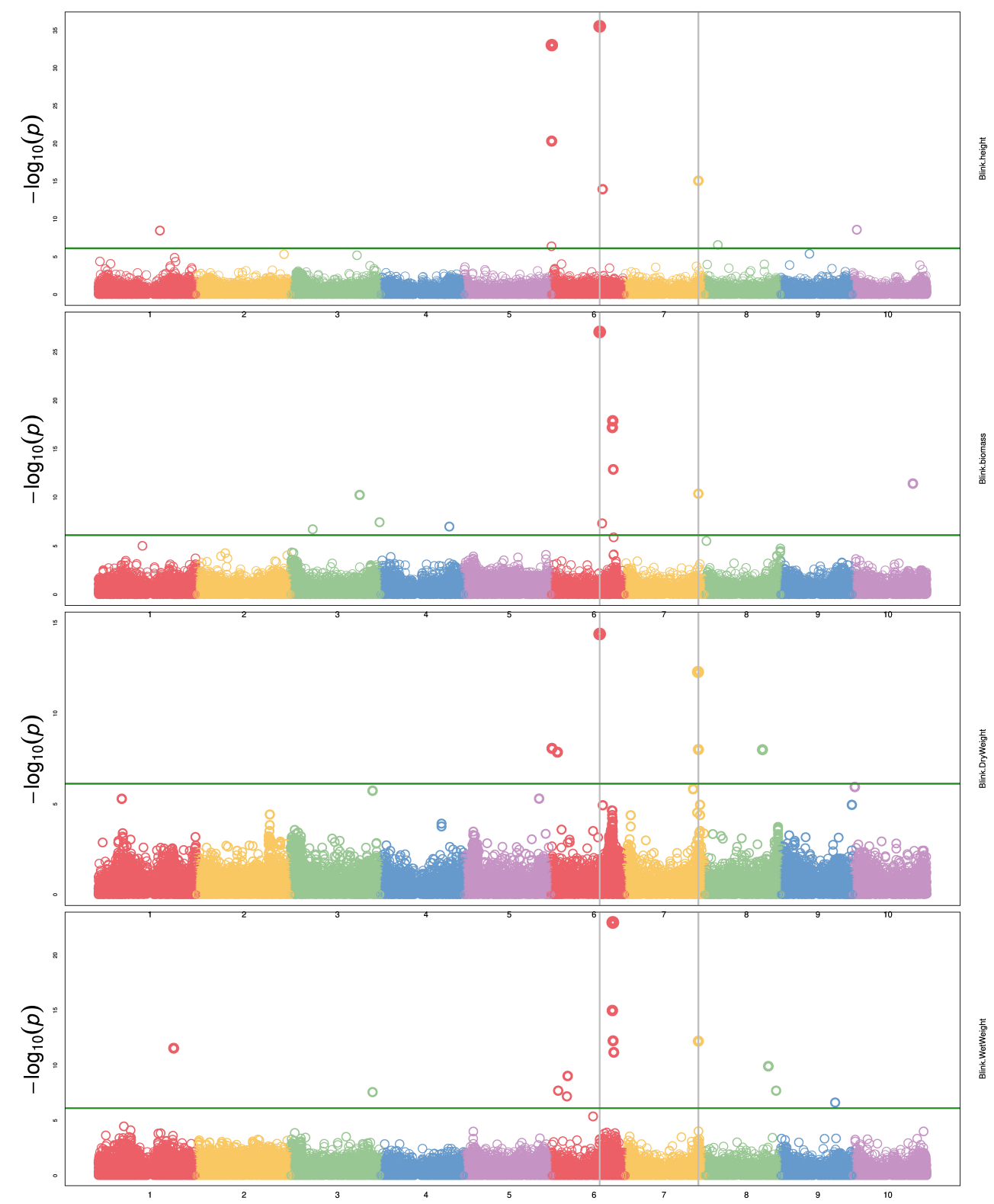

Figure 6. Manhattan plot of several agronomic traits using BLINK with maturity and DTH as covariates. (a) plant height, (b) biomass, (c) dry weight, and (d) wet weight. The green horizontal line represents the Bonferroni-corrected significance threshold. Vertical lines indicate SNPs common to multiple phenotypes. 
(a)

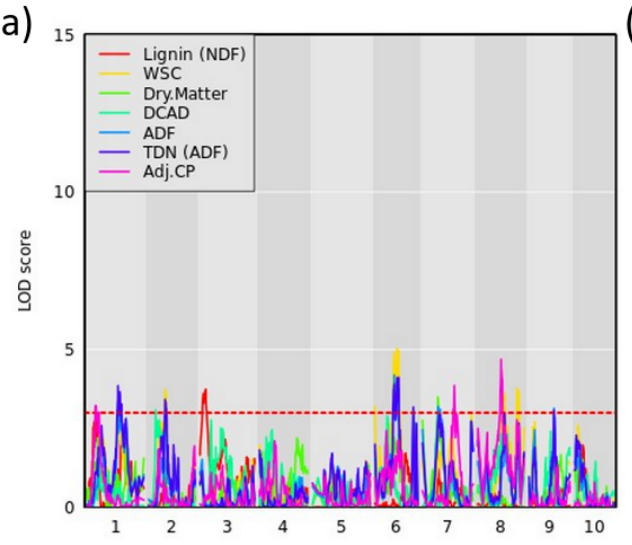

(c)

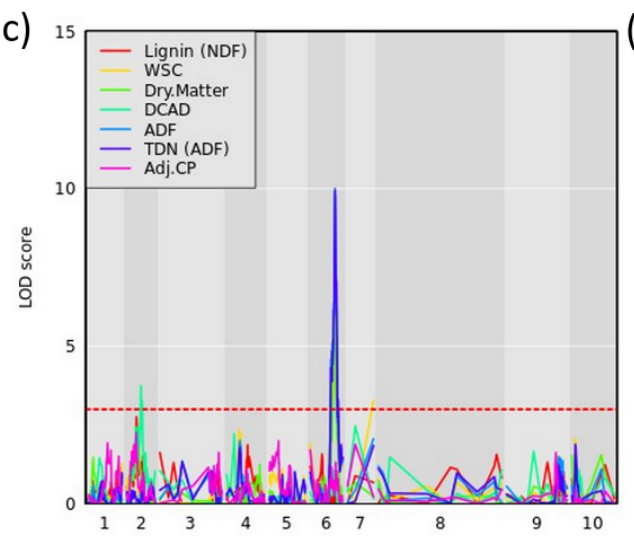

(b)

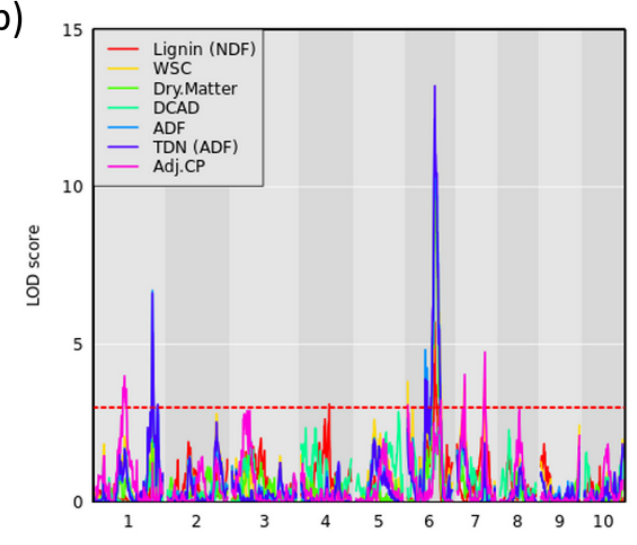

(c)

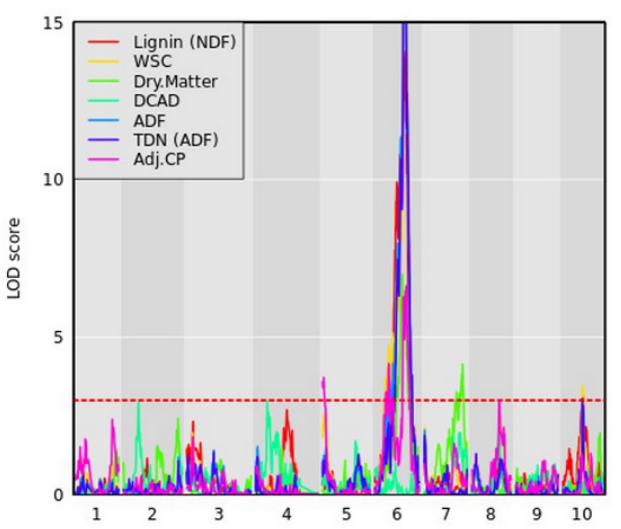

Figure 7. QTL mapping for compositional traits with maturity and DTH covariates where the red dashed line represents a logarithm of the odds (LOD) threshold of three in (a) PI22913, (b) PI297155, (c) PI508366, and (d) PI655972 RILs, which are sweet, grain, cellulosic, and forage recombinant populations, respectively. 
pleiotropic effects across multiple traits 86]. By using MV-LMMs on combinations of carbon-partitioning traits, we can better understand the interplay among these traits and predict the systemic effects of trait selection on the respective carbon sinks.

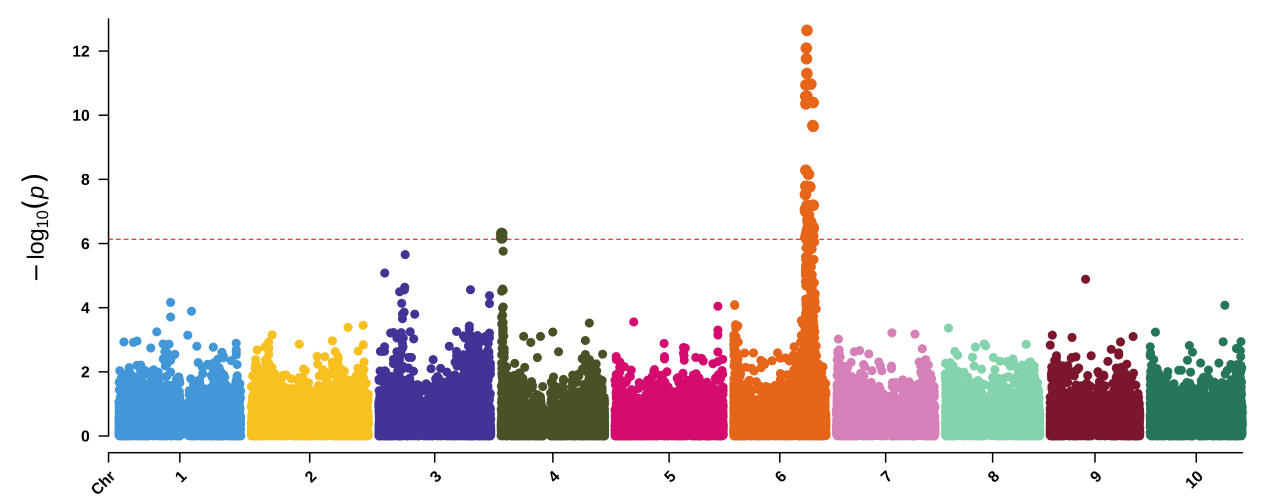

Figure 8. Manhattan plot of a MV-LMM using GEMMA with WSC and NDF as response variables and both maturity and DTH as covariates. The red-dashed, horizontal line represents the Bonferroni-corrected significance threshold.

Running WSC and NDF in a multivariate-response model with maturity and DTH as covariates approximates the dry matter phenotypic variance (Figure 2 , Figure 8). While only one locus has significant associations, the highly significant association on Chr6 occurs broadly from approximately 49.5 to $52 \mathrm{Mb}$ with the three most significant SNPs $(50,556,927 ; 50,558,124$; and 50,574,062). As noted from the QTL mapping results for WSC, this associated corresponds to the D locus. The most significant SNP (Chr6:50,558,124) exhibited considerable phenotypic variation across traits but contrasting effects for NDF and WSC for each allele (Figure S5-S6). The identity of the gene underlying this locus is believed to be a NAC transcription factor where recessive parents possess a premature stop codon in the NAC domain and were shown to exhibit lower lignin content but higher sugar and grain yields 84 similar to the relationship between NDF and WSC seen here. Running the same model but adding the top SNP as a covariate brings the peak on Chr4 above the significance threshold (Figures S7-S8), which is consistent with the dry matter LMM on Chr4 corresponding (Figure 10b). This SNP overlaps a QTL previously identified with dry matter growth rate, leaf appearance rate, [87, and stem circumference [76. Potential candidate genes in the region include two high-affinity nitrate transporter (NRT) genes

(Sobic.004G009400/Sobic.004G009500). In sorghum, increased expression of NRTs has been suggested to improve the efficiency at which inorganic and organic nitrogen is assimilated 88 and affect both the biomass and grain yield [89].

Using MV-LMM, we ran ash and lignin as response variables with maturity and DTH covariates (Figure 9), which may be roughly viewed as examining ADL (Figure 2). Though we do not have a direct measure of ADL for comparison, the significant loci are a subset of those found using ADF and NDF (Figures S9-S10) indicating the utility of MV-LMM and covariate models for compositional analyses.

By examining accumulation of soluble sugars in addition to lignocellulosic content and aggregate traits such as NDF, we may obtain a broader perspective of varying sink strength across the accessions. NDF represents a measure of the total lignocellulosic content. Lower lignin content and high lignocellulose production is preferred for efficient biofuel production [90. Using dry matter as the response variable, NDF was included in the LMM as a covariate to examine the effects of keeping NDF constant on the SNP 


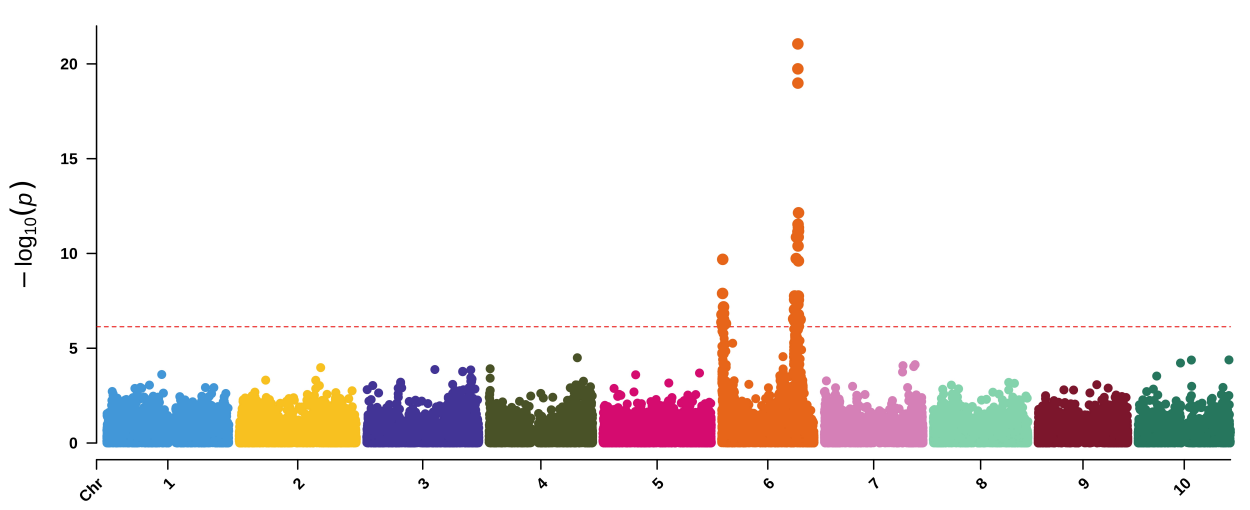

Figure 9. Manhattan plot of a MV-LMM using GEMMA with ash and lignin as response variables and both maturity and DTH as covariates. The red-dashed, horizontal line represents the Bonferroni-corrected significance threshold.

significance (Figure 10p). Biologically, this could be seen as running a model on the phenotypic variance of WSC. Similarly, using dry matter as the response variable with $\mathrm{ADF}$ as a covariate examines the phenotypic variance of hemicellulose and WSC (Figure 10.). By using covariates or multivariate-response models, the individual (i.e., WSC) or cumulative (i.e., NDF) phenotypic variance of compositional traits may be disentangled, and the relationships among traits may be more clearly distinguished.

Interestingly, the significantly associated SNPs are not identical between models such as a WSC LMM versus a dry matter response with NDF covariate LMM (Figure $10 p$, d) with composite traits such as dry matter often exhibiting more associated loci. As previously indicated, the integration of multi-scale phenotypes and multivariate models may be identifying emergent properties of these biological systems as some trait associations are not merely the sum of their parts [1,2, which further indicates the importance of running several different models that attempt to examine characteristics of a trait from multiple perspectives. The various dry matter models (Figure 10a-c; Figure S11) were also associated with a previously identified WSC locus containing a putative vacuolar iron transporter (VIT) on Chr4 [37]. It has been suggested that the candidate gene underlying this locus (Sobic.004G301500) may affect sugar accumulation either through neofunctionalization or via an iron-deficiency response 37. Though interestingly, the same LD block is also hit with adjusted crude protein as well as NEG (Figure S12-S13). Previous identification of a putative Dw4 locus identified from plant height GWAS also corresponds to this locus [81], and the locus has also been associated with increased total biomass and root biomass [91. Together, these results suggest a mechanism foundational to carbon accumulation underlying this locus, or the locus exhibits a pleiotropic effect on carbon accumulation or partitioning.

While the sorghum gene is classified as an iron transporter, further comparison with the Arabidopsis ortholog (AT3G43660) indicates a potential role in cellular manganese ion homeostasis (GO:0030026) 92 93. The CCC2-like domain of Sobic.004G301500 or one of the duplicate loci (Sobic.004G301600/Sobic.004G301650) therefore likely acts to transport manganese to vacuoles and maintain manganese homeostasis. As manganese serves to increase nitrogen assimilation [94, is a fundamental catalyst during the water-splitting reaction of photosystem II 95], and is necessary for respiration [96, a pivotal role in manganese homeostasis might better explain these associations. Further, since a role in manganese homeostasis has been described, these duplicated loci may instead demonstrate subfunctionalization followed by tissue-specific expression of one 
copy or the duplication may alter gene dosage and consequently modify some rate-limiting process. The association on Chr7 at approximately $59.5 \mathrm{Mb}$ found using dry matter with an ADF covariate, which is equivalent to looking at phenotypic variation due to WSC and hemicellulose, is Dw3 (Figure 10.).

To estimate the pleiotropic effects of variants across traits, we also performed a meta-analysis of SNP effects estimated using LMMs with an empirical Bayesian multivariate adaptive shrinkage approach that included results from nine traits 70. Over 150 variants exhibited strong associations across the nine traits with most associations occurring in chromosomes six and seven (Figure S14). While most associations occur within known loci including Dw3, Ma3, Ma6, and the D locus, a SNP on Chr10 colocalizes with previously identified locus for fresh stem weight and juice yield [97] as well as sucrose content 82. Taken together, these multivariate approaches highlight the pleiotropic effects of loci across the sorghum genome and support the importance of collecting peripherally related phenotypes to maximize carbon accumulation and partitioning.

\section{Discussion}

The diverse carbon-partitioning regimes of sorghum have the potential to provide valuable insights into the genetic control of carbon partitioning in grasses $[98$ from transport 38] to compartmentalization [99. Genes sensitive to carbohydrate concentration compose part of a highly conserved network necessary for cellular adjustment to nutrient availability and the partitioning of carbon among tissues and organs 100]. A holistic understanding of these processes requires multiscale phenotypes from molecule-specific quantification to anatomically aggregated measures of carbon. These multiscale metrics are necessary to accurately assess traits such as biomass where optical measures are typically poorly correlated with manually collected, macroscale phenotypes 3]. Orthogonal and partially correlated measures of diverse morphology assist in resolving functional questions of plant growth and development while simultaneously improving significant associations with functional genomic data. In conjunction with broad-scale phenotyping, multiparameter statistical approaches improve inferences through joint consideration of genomic and phenotypic measures 3 .

Here, we identified numerous putative loci that are associated with a variety of different phenotypes across different scales. The interactions between loci and locus pleiotropy hint at the underlying genetic architecture of these dynamic

carbon-partitioning traits. However, source and sink interactions can complicate the dissection of individual traits 33]. High-capacity, non-photosynthetic sinks can increase yield through sugar-responsive genes that mediate feed-forward loops that ultimately bolster systemic carbon accumulation 37,101]. Conversely, as seen here with the $D$ locus NAC transcription factor exhibiting reduced lignin content but increased sugar and grain yields 84, selection for some loci can result in a tradeoff between carbon regimes. The identification of these feedback mechanisms suggests that ongoing optimization of carbon allocation should simultaneously focus on improved source and sink strengths as a system of interconnected processes from nitrogen assimilation to photosynthetic efficiency $29,33,37$. Additionally, these findings indicate that sorghum yields (i.e., sugar, grain, forage, and biomass) may be further optimized to incorporate beneficial alleles from other sorghum types.

Using these models, we identified numerous candidate loci associated with carbon-partitioning traits using the CP-NAM. Several traits, such as WSC and biomass traits, shared associated loci supporting previous observations that selection for non-target, sink-related traits may collectively increase yields across carbon-partitioning regimes. Future studies should examine multi-trait, multi-environment data to further 

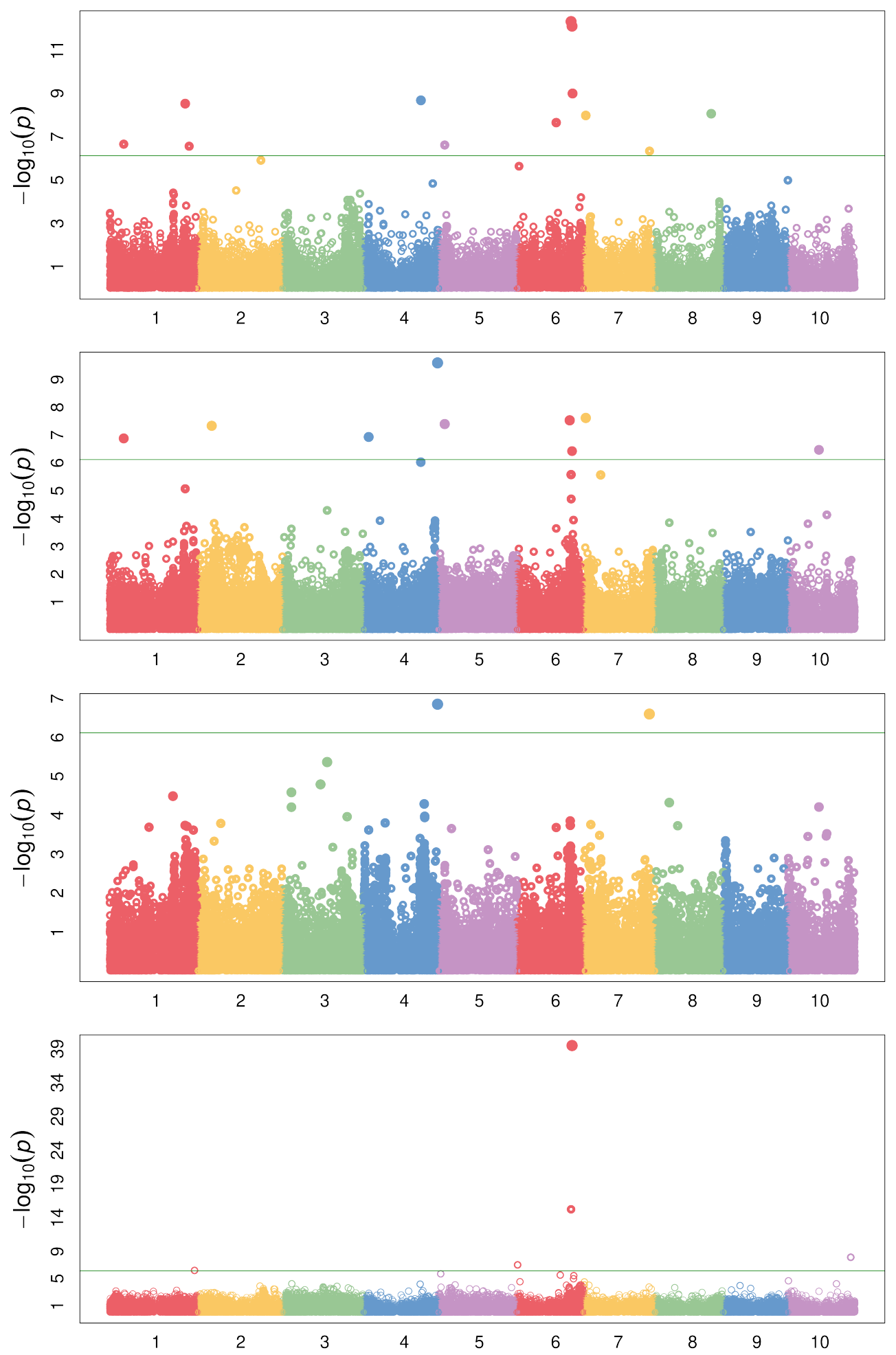

Figure 10. Manhattan plot of several compositional traits with different covariates following the design represented by Figure 1. (a) dry matter with maturity and DTH covariates, (b) dry matter with maturity, DTH, and NDF covariates, (c) dry matter with maturity, DTH, and ADF covariates, and (d) WSC with maturity and DTH covariates. The green horizontal line represents the Bonferroni-corrected significance threshold. 
extricate the environmental and genotype-by-environment effects on carbon-partitioning ${ }_{425}$ traits by leveraging the power of multiscale traits and MV-LMMs [102]. Additionally, breeders may consider collecting peripherally related traits as a means of understanding and maximizing carbon flow in their system as selection for carbon sinks is not a zero-sum relationship.

\section{Declarations}

\section{Authors' contributions}

JLB wrote the manuscript. JLB performed all computational analyses. AC, MM, NK, and SS managed field experiments. AC, KEJ, MM, NK, and SS collected phenotypes. JLB, SS, and SK conceptualized, developed, and implemented the study design.

\section{Availability of data and material}

Supplemental figures and tables are available with submission.

\section{Code availability}

Scripts are available on GitHub (https://github.com/jlboat/CP-NAM_2021) under MIT License.

\section{Conflicts of interest}

The authors declare no conflicts of interest.

\section{Consent for publication}

All authors reviewed and approved the final manuscript.

\section{Funding}

This project was funded in part by the U.S. Department of Energy's Advanced Research Project Agency award number DE-AR0001134. Any opinions, findings, conclusions, or recommendations expressed in this publication are those of the authors and do not necessarily reflect the views of the U.S. Department of Energy.

\section{Acknowledgments}

Computational analyses were performed on Clemson University's Palmetto Cluster, and we thank the staff who assisted with cluster and software maintenance.

\section{References}

1. H. P. Fischer, "Mathematical modeling of complex biological systems: from parts lists to understanding systems behavior," Alcohol Res. Health, vol. 31, no. 1, pp. 49-59, 2008.

2. B. Benes, K. Guan, M. Lang, S. P. Long, J. P. Lynch, A. Marshall-Colón, B. Peng, J. Schnable, L. J. Sweetlove, and M. J. Turk, "Multiscale computational models can guide experimentation and targeted measurements for crop improvement," Plant J., vol. 103, no. 1, pp. 21-31, Jul. 2020. 
3. M. Eberius and J. Lima-Guerra, "High-throughput plant phenotyping - data acquisition, transformation, and analysis," in Bioinformatics. New York, NY: Springer New York, 2009, pp. 259-278.

4. A. C. Chipanshi, R. Chanda, and O. Totolo, "Vulnerability assessment of the maize and sorghum crops to climate change in botswana," Clim. Change, vol. 61, no. 3, pp. 339-360, Dec. 2003.

5. J. Knox, T. Hess, A. Daccache, and T. Wheeler, "Climate change impacts on crop productivity in africa and south asia," Environ. Res. Lett., vol. 7, no. 3, p. 034032, Sep. 2012.

6. M. N. Meki, A. R. Kemanian, S. R. Potter, J. M. Blumenthal, J. R. Williams, and T. J. Gerik, "Cropping system effects on sorghum grain yield, soil organic carbon, and global warming potential in central and south texas," Agric. Syst., vol. 117, pp. 19-29, May 2013.

7. M. Druille, A. S. Williams, M. Torrecillas, S. Kim, N. Meki, and J. R. Kiniry, "Modeling climate warming impacts on grain and forage sorghum yields in argentina," Agronomy (Basel), vol. 10, no. 7, p. 964, Jul. 2020.

8. W. K. Michener, E. R. Blood, K. L. Bildstein, M. M. Brinson, and L. R. Gardner, "Climate change, hurricanes and tropical storms, and rising sea level in coastal wetlands," Ecol. Appl., vol. 7, no. 3, pp. 770-801, Aug. 1997.

9. M. J. Ottman, B. A. Kimball, P. J. Pinter, G. W. Wall, R. L. Vanderlip, S. W. Leavitt, R. L. LaMorte, A. D. Matthias, and T. J. Brooks, "Elevated CO2 increases sorghum biomass under drought conditions," New Phytol., vol. 150, no. 2, pp. 261-273, May 2001.

10. E. Pennisi, "Plant genetics. how sorghum withstands heat and drought," Science, vol. 323, no. 5914, p. 573, Jan. 2009.

11. K. Yan, P. Chen, H. Shao, L. Zhang, and G. Xu, "Effects of short-term high temperature on photosynthesis and photosystem II performance in sorghum," $J$. Agron. Crop Sci., vol. 197, no. 5, pp. 400-408, Oct. 2011.

12. D. B. Lobell and C. B. Field, "Global scale climate-crop yield relationships and the impacts of recent warming," Environ. Res. Lett., vol. 2, no. 1, p. 014002, Mar. 2007.

13. V. G. Kakani, J. C. V. Vu, L. H. Allen, and K. J. Boote, "Leaf photosynthesis and carbohydrates of $\mathrm{CO} 2$-enriched maize and grain sorghum exposed to a short period of soil water deficit during vegetative development," J. Plant Physiol., vol. 168, no. 18, pp. 2169-2176, Dec. 2011.

14. W. Zegada-Lizarazu, A. Zatta, and A. Monti, "Water uptake efficiency and above- and belowground biomass development of sweet sorghum and maize under different water regimes," Plant Soil, vol. 351, no. 1-2, pp. 47-60, Feb. 2012.

15. T. van der Weijde, C. L. Alvim Kamei, A. F. Torres, W. Vermerris, O. Dolstra, R. G. F. Visser, and L. M. Trindade, "The potential of C4 grasses for cellulosic biofuel production," Front. Plant Sci., vol. 4, p. 107, May 2013. 
16. H. W. Fischer, N. L. N. Reddy, and M. L. S. Rao, "Can more drought resistant crops promote more climate secure agriculture? prospects and challenges of millet cultivation in ananthapur, andhra pradesh," World Dev. Perspect., vol. 2, pp. 5-10, Jun. 2016.

17. E. A. Heaton, F. G. Dohleman, and S. P. Long, "Meeting US biofuel goals with less land: the potential of miscanthus," Glob. Chang. Biol., vol. 14, no. 9, pp. 2000-2014, Sep. 2008.

18. K. David and A. J. Ragauskas, "Switchgrass as an energy crop for biofuel production: A review of its ligno-cellulosic chemical properties," Energy Environ. Sci., vol. 3, no. 9, pp. 1182-1190, 2010.

19. S. N. Olson, K. Ritter, W. Rooney, A. Kemanian, B. A. McCarl, Y. Zhang, S. Hall, D. Packer, and J. Mullet, "High biomass yield energy sorghum: developing a genetic model for C4 grass bioenergy crops," pp. 640-655, 2012.

20. A. Monti, Switchgrass: A Valuable Biomass Crop for Energy. London: Springer Science \& Business Media, Mar. 2012.

21. N. Brosse, A. Dufour, X. Meng, Q. Sun, and A. Ragauskas, "Miscanthus: a fast-growing crop for biofuels and chemicals production," Biofuels Bioprod. Biorefin., vol. 6, no. 5, pp. 580-598, Sep. 2012.

22. J. Mullet, D. Morishige, R. McCormick, S. Truong, J. Hilley, B. McKinley, R. Anderson, S. N. Olson, and W. Rooney, "Energy sorghum-a genetic model for the design of C4 grass bioenergy crops," pp. 3479-3489, 2014.

23. N. C. Carpita and M. C. McCann, "Maize and sorghum: genetic resources for bioenergy grasses," Trends Plant Sci., vol. 13, no. 8, pp. 415-420, Aug. 2008.

24. P. V. V. Prasad, J. C. V. Vu, K. J. Boote, and L. H. Allen, "Enhancement in leaf photosynthesis and upregulation of rubisco in the $\mathrm{C} 4$ sorghum plant at elevated growth carbon dioxide and temperature occur at early stages of leaf ontogeny," Funct. Plant Biol., vol. 36, no. 9, pp. 761-769, Sep. 2009.

25. J. C. Gardner, J. W. Maranville, and E. T. Paparozzi, "Nitrogen use efficiency among diverse sorghum cultivars," Crop Sci., vol. 34, no. 3, pp. 728-733, May 1994.

26. C. S. Byrt, C. P. L. Grof, and R. T. Furbank, "C4 plants as biofuel feedstocks: Optimising biomass production and feedstock quality from a lignocellulosic PerspectiveFree access," J. Integr. Plant Biol., vol. 53, no. 2, pp. 120-135, Feb. 2011.

27. B. Bhattarai, S. Singh, C. P. West, and R. Saini, "Forage potential of pearl millet and forage sorghum alternatives to corn under the Water-Limiting conditions of the texas high plains: A review," Crop, Forage 8 Turfgrass Management, vol. 5, no. 1, pp. 1-12, 2019.

28. W. L. Rooney, J. Blumenthal, B. Bean, and J. E. Mullet, "Designing sorghum as a dedicated bioenergy feedstock," Biofuels Bioprod. Biorefin., vol. 1, no. 2, pp. 147-157, Oct. 2007.

29. J. L. Boatwright, Z. W. Brenton, R. E. Boyles, S. Sapkota, M. T. Myers, K. E. Jordan, S. M. Dale, N. Shakoor, E. A. Cooper, G. P. Morris, and S. Kresovich, "Genetic characterization of a sorghum bicolor multiparent mapping population emphasizing carbon-partitioning dynamics," G3, vol. 11, no. 4, Apr. 2021. 
30. G. P. Morris, P. Ramu, S. P. Deshpande, C. T. Hash, T. Shah, H. D. Upadhyaya, O. Riera-Lizarazu, P. J. Brown, C. B. Acharya, S. E. Mitchell, and Others, "Population genomic and genome-wide association studies of agroclimatic traits in sorghum," Proceedings of the National Academy of Sciences, vol. 110, no. 2, pp. 453-458, 2013.

31. M. Calviño and J. Messing, "Sweet sorghum as a model system for bioenergy crops," Curr. Opin. Biotechnol., vol. 23, no. 3, pp. 323-329, Jun. 2012.

32. Y. L. Zhao, A. Dolat, Y. Steinberger, X. Wang, A. Osman, and G. H. Xie, "Biomass yield and changes in chemical composition of sweet sorghum cultivars grown for biofuel," Field Crops Res., vol. 111, no. 1-2, pp. 55-64, 2009.

33. Z. W. Brenton, E. A. Cooper, M. T. Myers, R. E. Boyles, N. Shakoor, K. J. Zielinski, B. L. Rauh, W. C. Bridges, G. P. Morris, and S. Kresovich, "A genomic resource for the development, improvement, and exploitation of sorghum for bioenergy," Genetics, vol. 204, no. 1, pp. 21-33, Sep. 2016.

34. R. F. McCormick, S. K. Truong, A. Sreedasyam, J. Jenkins, S. Shu, D. Sims, M. Kennedy, M. Amirebrahimi, B. D. Weers, B. McKinley, A. Mattison, D. T. Morishige, J. Grimwood, J. Schmutz, and J. E. Mullet, "The sorghum bicolor reference genome: improved assembly, gene annotations, a transcriptome atlas, and signatures of genome organization," Plant J., vol. 93, no. 2, pp. 338-354, Jan. 2018.

35. S. Peng, D. R. Krieg, and F. S. Girma, "Leaf photosynthetic rate is correlated with biomass and grain production in grain sorghum lines," pp. 1-7, 1991.

36. H. A. Qazi, S. Paranjpe, and S. Bhargava, "Stem sugar accumulation in sweet sorghum - activity and expression of sucrose metabolizing enzymes and sucrose transporters," J. Plant Physiol., vol. 169, no. 6, pp. 605-613, Apr. 2012.

37. Z. W. Brenton, B. T. Juengst, E. A. Cooper, M. T. Myers, K. E. Jordan, S. M. Dale, J. C. Glaubitz, X. Wang, R. E. Boyles, E. L. Connolly, and S. Kresovich, "Species-Specific duplication event associated with elevated levels of nonstructural carbohydrates in sorghum bicolor," G3, vol. 10, no. 5, pp. 1511-1520, May 2020.

38. R. J. Milne, C. S. Byrt, J. W. Patrick, and C. P. L. Grof, "Are sucrose transporter expression profiles linked with patterns of biomass partitioning in sorghum phenotypes?" Front. Plant Sci., vol. 4, p. 223, Jun. 2013.

39. E. A. Cooper, Z. W. Brenton, B. S. Flinn, J. Jenkins, S. Shu, D. Flowers, F. Luo, Y. Wang, P. Xia, K. Barry, C. Daum, A. Lipzen, Y. Yoshinaga, J. Schmutz, C. Saski, W. Vermerris, and S. Kresovich, "A new reference genome for sorghum bicolor reveals high levels of sequence similarity between sweet and grain genotypes: implications for the genetics of sugar metabolism," $B M C$ Genomics, vol. 20, no. 1, p. 420, May 2019.

40. D. M. Vietor and F. R. Miller, "Assimilation, partitioning, and nonstructural carbohydrates in sweet compared with grain sorghum," p. 1109, 1990.

41. M. Billings, "Biomass sorghum and sweet sorghum data gathering report," WEA Crop Insurance. USDA-RMA, CTOR: Jaime, 2015.

42. B. George-Jaeggli, D. R. Jordan, E. J. van Oosterom, and G. L. Hammer, "Decrease in sorghum grain yield due to the dw3 dwarfing gene is caused by reduction in shoot biomass," Field Crops Res., vol. 124, no. 2, pp. 231-239, Nov. 2011. 
43. S. C. Murray, A. Sharma, W. L. Rooney, P. E. Klein, J. E. Mullet, S. E. Mitchell, and S. Kresovich, "Genetic improvement of sorghum as a biofuel feedstock: I. QTL for stem sugar and grain nonstructural carbohydrates," Crop Sci., vol. 48, no. 6, pp. 2165-2179, 2008.

44. S. Kresovich, F. R. Miller, R. L. Monk, R. E. Dominy, and D. M. Broadhead, "Registration of 'grassl' sweet sorghum," pp. 194-195, 1988.

45. J. Yu and E. S. Buckler, "Genetic association mapping and genome organization of maize," Curr. Opin. Biotechnol., vol. 17, no. 2, pp. 155-160, 2006.

46. E. Schubert and P. J. Rousseeuw, "Faster k-medoids clustering: Improving the PAM, CLARA, and CLARANS algorithms," in Similarity Search and Applications, ser. Lecture notes in computer science. Cham: Springer International Publishing, 2019, pp. 171-187.

47. B. Guo, D. A. Sleper, and W. D. Beavis, "Nested association mapping for identification of functional markers," Genetics, vol. 186, no. 1, pp. 373-383, Sep. 2010.

48. M. Viel, F. Collet, S. Prétot, and C. Lanos, "Hemp-straw composites: Gluing study and multi-physical characterizations," Materials (Basel), vol. 12, no. 8, p. 1199, Apr. 2019.

49. W. Kruijer, M. P. Boer, M. Malosetti, P. J. Flood, B. Engel, R. Kooke, J. J. B. Keurentjes, and F. A. van Eeuwijk, "Marker-based estimation of heritability in immortal populations," Genetics, vol. 199, no. 2, pp. 379-398, Feb. 2015.

50. D. Bates, M. Mächler, B. Bolker, and S. Walker, "Fitting linear Mixed-Effects models using lme4," J. Stat. Softw., vol. 67, no. 1, pp. 1-48, 2015.

51. M. Waskom, "seaborn: statistical data visualization," J. Open Source Softw., vol. 6, no. 60, p. 3021, Apr. 2021.

52. W. McKinney, "Data structures for statistical computing in python," in Proceedings of the 9th Python in Science Conference, ser. Proceedings of the Python in Science Conference. Austin: SciPy, 2010, pp. 56-61.

53. G. Van Rossum and F. L. Drake, Introduction to Python 3: (Python Documentation Manual Part 1). California: CreateSpace Independent Publishing Platform, Mar. 2009.

54. J. C. Glaubitz, T. M. Casstevens, F. Lu, J. Harriman, R. J. Elshire, Q. Sun, and E. S. Buckler, "TASSEL-GBS: a high capacity genotyping by sequencing analysis pipeline," PLoS One, vol. 9, no. 2, p. e90346, 2014.

55. B. L. Browning, Y. Zhou, and S. R. Browning, "A One-Penny imputed genome from Next-Generation reference panels," Am. J. Hum. Genet., vol. 103, no. 3, pp. 338-348, Sep. 2018.

56. D. D. Kosambi, "The estimation of map distances from recombination values," in D.D. Kosambi: Selected Works in Mathematics and Statistics,

R. Ramaswamy, Ed. New Delhi: Springer India, 2016, pp. 125-130.

57. K. W. Broman, D. M. Gatti, P. Simecek, N. A. Furlotte, P. Prins, Ś. Sen, B. S. Yandell, and G. A. Churchill, "R/qtl2: Software for mapping quantitative trait loci with High-Dimensional data and multiparent populations," Genetics, vol. 211, no. 2, pp. 495-502, Feb. 2019. 
58. C. S. Haley and S. A. Knott, "A simple regression method for mapping quantitative trait loci in line crosses using flanking markers," Heredity (Edinb.), vol. 69, no. 4, pp. 315-324, Oct. 1992.

59. K. W. Broman and S. Sen, A Guide to QTL Mapping with R/qtl. Springer, 2009, vol. 46.

60. E. G. King and A. D. Long, "The beavis effect in next-generation mapping panels in drosophila melanogaster," G3, vol. 7, no. 6, pp. 1643-1652, Jun. 2017.

61. X. Zhou and M. Stephens, "Efficient multivariate linear mixed model algorithms for genome-wide association studies," Nat. Methods, vol. 11, no. 4, p. 407, 2014.

62. A. E. Lipka, F. Tian, Q. Wang, J. Peiffer, M. Li, P. J. Bradbury, M. A. Gore, E. S. Buckler, and Z. Zhang, "GAPIT: genome association and prediction integrated tool," Bioinformatics, vol. 28, no. 18, pp. 2397-2399, 2012.

63. J. Wang and Z. Zhang, "GAPIT version 3: boosting power and accuracy for genomic association and prediction," BioRxiv, 2020.

64. X. Zhou, P. Carbonetto, and M. Stephens, "Polygenic modeling with bayesian sparse linear mixed models," PLoS Genet., vol. 9, no. 2, p. e1003264, 2013.

65. M. Huang, X. Liu, Y. Zhou, R. M. Summers, and Z. Zhang, "BLINK: a package for the next level of genome-wide association studies with both individuals and markers in the millions," 2019.

66. S. Purcell, B. Neale, K. Todd-Brown, L. Thomas, M. A. R. Ferreira, D. Bender, J. Maller, P. Sklar, P. I. W. De Bakker, M. J. Daly, and Others, "PLINK: a tool set for whole-genome association and population-based linkage analyses," Am. J. Hum. Genet., vol. 81, no. 3, pp. 559-575, 2007.

67. P. M. VanRaden, "Efficient methods to compute genomic predictions," J. Dairy Sci., vol. 91, no. 11, pp. 4414-4423, 2008.

68. S. Sapkota, R. Boyles, E. Cooper, Z. Brenton, M. Myers, and S. Kresovich, "Impact of sorghum racial structure and diversity on genomic prediction of grain yield components," Crop Sci., Jan. 2020.

69. M. Stephens, "False discovery rates: a new deal," Biostatistics, vol. 18, no. 2, pp. 275-294, 2017.

70. S. M. Urbut, G. Wang, P. Carbonetto, and M. Stephens, "Flexible statistical methods for estimating and testing effects in genomic studies with multiple conditions," Nature genetics, vol. 51, no. 1, pp. 187-195, 2019.

71. A. H. MacQueen, J. W. White, R. Lee, J. M. Osorno, J. Schmutz, P. N. Miklas, J. Myers, P. E. McClean, and T. E. Juenger, "Genetic associations in four decades of multienvironment trials reveal agronomic trait evolution in common bean," Genetics, vol. 215, no. 1, pp. 267-284, 2020.

72. D. Ortiz, J. Hu, and M. G. Salas Fernandez, "Genetic architecture of photosynthesis in sorghum bicolor under non-stress and cold stress conditions," J. Exp. Bot., vol. 68, no. 16, pp. 4545-4557, Jul. 2017.

73. E. S. Mace, S. Tai, E. K. Gilding, Y. Li, P. J. Prentis, L. Bian, B. C. Campbell, W. Hu, D. J. Innes, X. Han, and Others, "Whole-genome sequencing reveals untapped genetic potential in africa's indigenous cereal crop sorghum," Nat. Commun., vol. 4, p. 2320, 2013. 
74. S. R. Marla, G. Burow, R. Chopra, C. Hayes, M. O. Olatoye, T. Felderhoff, Z. Hu, R. Raymundo, R. Perumal, and G. P. Morris, "Genetic architecture of chilling tolerance in sorghum dissected with a nested association mapping population," pp. 4045-4057, 2019.

75. S. Yang, R. L. Murphy, D. T. Morishige, P. E. Klein, W. L. Rooney, and J. E. Mullet, "Sorghum phytochrome B inhibits flowering in long days by activating expression of SbPRR37 and SbGHD7, repressors of SbEHD1, SbCN8 and SbCN12," PLoS One, vol. 9, no. 8, p. e105352, Aug. 2014.

76. J. Zhao, M. B. Mantilla Perez, J. Hu, and M. G. Salas Fernandez, "Genome-wide association study for nine plant architecture traits in sorghum," Plant Genome, vol. 9, no. 2, Jul. 2016.

77. S. Bouchet, M. O. Olatoye, S. R. Marla, R. Perumal, T. Tesso, J. Yu, M. Tuinstra, and G. P. Morris, "Increased power to dissect adaptive traits in global sorghum diversity using a nested association mapping population," Genetics, vol. 206, no. 2, pp. 573-585, Jun. 2017.

78. W. Kong, C. Kim, D. Zhang, H. Guo, X. Tan, H. Jin, C. Zhou, L.-S. Shuang, V. Goff, U. Sezen, G. Pierce, R. Compton, C. Lemke, J. Robertson, L. Rainville, S. Auckland, and A. H. Paterson, "Genotyping by sequencing of 393 sorghum bicolor BTx623 $\times$ IS3620C recombinant inbred lines improves sensitivity and resolution of QTL detection," G3 (Bethesda), vol. 8, no. 8, pp. 2563-2572, Jul. 2018.

79. R. L. Murphy, D. T. Morishige, J. A. Brady, W. L. Rooney, S. Yang, P. E. Klein, and J. E. Mullet, "Ghd7 ( ma 6 ) represses sorghum flowering in long days: Ghd7 alleles enhance biomass accumulation and grain production," Plant Genome, vol. 7, no. 2, p. lantgenome2013.11.0040, Jul. 2014.

80. J. L. Hilley, B. D. Weers, S. K. Truong, R. F. McCormick, A. J. Mattison, B. A. McKinley, D. T. Morishige, and J. E. Mullet, "Sorghum dw2 encodes a protein kinase regulator of stem internode length," Sci. Rep., vol. 7, no. 1, p. 4616, Jul. 2017.

81. X. Li, X. Li, E. Fridman, T. T. Tesso, and J. Yu, "Dissecting repulsion linkage in the dwarfing gene dw3 region for sorghum plant height provides insights into heterosis," Proc. Natl. Acad. Sci. U. S. A., vol. 112, no. 38, pp. 11823-11828, Sep. 2015.

82. K. B. Ritter, D. R. Jordan, S. C. Chapman, I. D. Godwin, E. S. Mace, and C. Lynne McIntyre, "Identification of QTL for sugar-related traits in a sweet $\times$ grain sorghum (sorghum bicolor l. moench) recombinant inbred population," Mol. Breed., vol. 22, no. 3, pp. 367-384, Oct. 2008.

83. P. S. Burks, C. M. Kaiser, E. M. Hawkins, and P. J. Brown, "Genomewide association for sugar yield in sweet sorghum," Crop Sci., vol. 55, no. 5, pp. 2138-2148, Sep. 2015.

84. J. Xia, Y. Zhao, P. Burks, M. Pauly, and P. J. Brown, "A sorghum NAC gene is associated with variation in biomass properties and yield potential," Plant Direct, vol. 2, no. 7, p. e00070, Jul. 2018.

85. B. R. Rice, S. B. Fernandes, and A. E. Lipka, "Multi-Trait genome-wide association studies reveal loci associated with maize inflorescence and leaf architecture," Plant Cell Physiol., 2020. 
86. R. V. Mural, M. Grzybowski, C. Miao, A. Damke, S. Sapkota, R. E. Boyles, M. G. Salas Fernandez, P. S. Schnable, B. Sigmon, S. Kresovich, and J. C. Schnable, "Meta-Analysis identifies pleiotropic loci controlling phenotypic trade-offs in sorghum," Genetics, vol. 218, no. 3, Jun. 2021.

87. K. Fiedler, W. A. Bekele, R. Duensing, S. Gründig, R. Snowdon, H. Stützel, A. Zacharias, and R. Uptmoor, "Genetic dissection of temperature-dependent sorghum growth during juvenile development," Züchter Genet. Breed. Res., vol. 127, no. 9, pp. 1935-1948, Sep. 2014.

88. M. Gelli, Y. Duo, A. R. Konda, C. Zhang, D. Holding, and I. Dweikat, "Identification of differentially expressed genes between sorghum genotypes with contrasting nitrogen stress tolerance by genome-wide transcriptional profiling," BMC Genomics, vol. 15, p. 179, Mar. 2014.

89. M. Gelli, A. R. Konda, K. Liu, C. Zhang, T. E. Clemente, D. R. Holding, and I. M. Dweikat, "Validation of QTL mapping and transcriptome profiling for identification of candidate genes associated with nitrogen stress tolerance in sorghum," BMC Plant Biol., vol. 17, no. 1, Dec. 2017.

90. S.-J. Jung, S.-H. Kim, and I.-M. Chung, "Comparison of lignin, cellulose, and hemicellulose contents for biofuels utilization among 4 types of lignocellulosic crops," Biomass Bioenergy, vol. 83, pp. 322-327, Dec. 2015.

91. N. Moghimi, J. S. Desai, R. Bheemanahalli, S. M. Impa, A. R. Vennapusa, D. Sebela, R. Perumal, C. J. Doherty, and S. V. K. Jagadish, "New candidate loci and marker genes on chromosome 7 for improved chilling tolerance in sorghum," J. Exp. Bot., vol. 70, no. 12, pp. 3357-3371, Jun. 2019.

92. J. Gollhofer, C. Schläwicke, N. Jungnick, W. Schmidt, and T. J. Buckhout, "Members of a small family of nodulin-like genes are regulated under iron deficiency in roots of arabidopsis thaliana," Plant Physiol. Biochem., vol. 49, no. 5, pp. 557-564, May 2011.

93. T. Z. Berardini, L. Reiser, D. Li, Y. Mezheritsky, R. Muller, E. Strait, and E. Huala, "The arabidopsis information resource: Making and mining the "gold standard" annotated reference plant genome," Genesis, vol. 53, no. 8, pp. 474-485, Aug. 2015.

94. E. Przemeck and B. Schrader, "The effect of manganese nutrition on nitrogen assimilation in roots," in Structure and Function of Plant Roots. Dordrecht: Springer Netherlands, 1981, pp. 123-127.

95. W. W. Fischer, J. Hemp, and J. E. Johnson, "Manganese and the evolution of photosynthesis," Orig. Life Evol. Biosph., vol. 45, no. 3, pp. 351-357, Sep. 2015.

96. S. Alejandro, S. Höller, B. Meier, and E. Peiter, "Manganese in plants: From acquisition to subcellular allocation," Front. Plant Sci., vol. 11, p. 300, Mar. 2020 .

97. P. Lv, G. Ji, Y. Han, S. Hou, S. Li, X. Ma, R. Du, and G. Liu, "Association analysis of sugar yield-related traits in sorghum [sorghum bicolor (l.)]," Euphytica, vol. 193, no. 3, pp. 419-431, 2013.

98. D. M. Braun and T. L. Slewinski, "Genetic control of carbon partitioning in grasses: roles of sucrose transporters and tie-dyed loci in phloem loading," Plant Physiol., vol. 149, no. 1, pp. 71-81, Jan. 2009. 
99. R. T. Furbank and S. Kelly, "Finding the C4 sweet spot: cellular compartmentation of carbohydrate metabolism in C4 photosynthesis," J. Exp. Bot., Jun. 2021.

100. K. E. Koch, "Carbohydrate-modulated gene expression in plants," Annu. Rev. Plant Physiol. Plant Mol. Biol., vol. 47, no. 1, pp. 509-540, Jun. 1996.

101. S. Bihmidine, C. T. Hunter, 3rd, C. E. Johns, K. E. Koch, and D. M. Braun, "Regulation of assimilate import into sink organs: update on molecular drivers of sink strength," Front. Plant Sci., vol. 4, p. 177, Jun. 2013.

102. G. Covarrubias-Pazaran, "Genome-Assisted prediction of quantitative traits using the R package sommer," PLoS One, 2016. 